\title{
Observation of dynamic 3D motion of nanoparticles combined with 4D- STEM orientation and phase map in Liquid-Cell STEM microscopy
}

Alejandro Gomez-Perez ${ }^{1}$, Athanassios Galanis ${ }^{1}$, Partha Das ${ }^{1}$, Stavros Nicolopoulos ${ }^{1}$ and Arnaud Demortière $^{2}$

${ }^{1}$ NanoMEGAS SPRL, Brussels, Belgium, Brussels, Belgium, ${ }^{2}$ Laboratoire de Réactivité et de Chimie des solides (LRCS), Université de Picardie Jules Verne, CNRS UMR 7314, 33 rue Saint Leu, 80039 Amiens Cedex, France, United States

Direct observation of 3D motion of nanoparticles is of critical importance for the study of interparticle interactions and for in situ colloidal self-assembly using Liquid Cell (LC). Measuring nanoparticle dynamics with high spatial and temporal resolution opens potential for dynamic studies in LC as well as interaction between particles and substrates. For this study we have used TEM based automated 4D(precession) STEM crystallographic mapping techniques (ASTAR) [1] to study phase \& orientation mapping (down to $1-5 \mathrm{~nm}$ scale) of nanoparticles as they dynamically interact in situ at different time scales within the liquid.

For our experiments we have used a Liquid cell (K-Kit from Ma-Tek company) and standard gold multidomain nanoparticles of $60 \mathrm{~nm}$ diameter. The LC spacer (distance of two $\mathrm{Si}_{3} \mathrm{~N}_{4}$ membranes of $30 \mathrm{~nm}$ that seal and encapsulate the liquid) was $200 \mathrm{~nm}$. Using a Tecnai G20 ( $\left.\mathrm{LaB}_{6}\right)$ FEI-TFS microscope interfaced with ASTAR, we collected two sets of precession electron diffraction patterns (PED) of the particles while the beam was scanned over an area of $360 \times 360 \mathrm{~nm}$ ( $1 \mathrm{st}$ data sets for $\mathrm{t}=0 \mathrm{sec}, \mathrm{t}=5 \mathrm{~min}$ and $\mathrm{t}=10 \mathrm{~min}$, step size $6 \mathrm{~nm}$, scanning time $3 \mathrm{~min}$ per scan) and $220 \times 220 \mathrm{~nm}$ (2nd data series, for $\mathrm{t}=0 \mathrm{sec}, \mathrm{t}=60 \mathrm{sec}$ and $\mathrm{t}=120 \mathrm{sec}$, step size $11 \mathrm{~nm}$, scanning time $20 \mathrm{sec}$ per scan). PED patterns (precession angle $0.4^{\circ}$ ) were noise free as were collected using latest generation Medipix III direct detection camera (ASI) with a rate of $20 \mathrm{fps}, 12 \mathrm{bit}$.

We can observe from the first data sets in Fig.1, an agglomeration of Au particles with corresponding texture $\&$ orientation map generated from all particles. At different time lapses $(0,5 \mathrm{~min}, 10 \mathrm{~min})$ the four $\mathrm{Au}$ nanoparticles are in close contact and show similar orientation map (that reflect in detail their domain structure) with time (0-10 $\mathrm{min})$, while an individual nanoparticle starts its own motion within the liquid, moving away from the group of four particles, while it rotates with time (as clearly seen through its change of orientation map with time). Noise free ED patterns (where ED patterns corresponds to particles highlighted by white arrow in Fig 1) as well subtle changes observed on the texture maps, reveal very precisely those orientation /rotational changes of the nanoparticles within time.

In the second data sets in Fig.2 an orientation map of different Au nanoparticle agglomeration (where texture map is generated from all particles) can be observed at various time lapses $(0,60$ and $120 \mathrm{sec})$. Although particles do not move substantially within this time frame, subtle orientation changes are observed (from the change in colour in ASTAR orientation maps as well from slight changes on the texture maps). Those changes may be indicative of local orientation /rotational changes and/or particle movement along the z-direction. As the gold particles are diffusing inside the LC, distinct particles may appear blurred to various extent indicating that they can be at different heights within the LC, where such particle blurring may give us an indication of their position within the LC [2]. 
Although plots of individual particle trajectories are difficult to be used as a rigorous basis to determine the nature of their motion (Brownian or not), rotational hydrodynamics of nanoparticles can be effectively probed with high resolution within LC using 4D-STEM ASTAR techniques in combination with Direct Detection noise-free cameras. This type of studies may have an important impact in the near future for the study of materials in liquid environments.

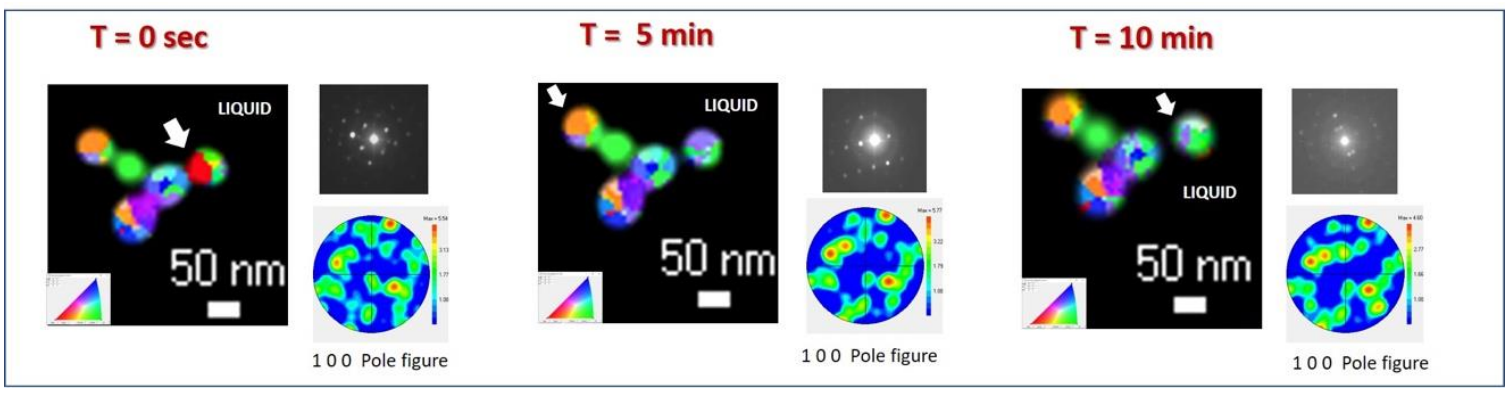

Figure 1. ASTAR orientation map of $360 \mathrm{~nm}$ x $360 \mathrm{~nm}$ (scan time $3 \mathrm{~min}$ ) by Medipix III detector, $20 \mathrm{fps}$, 12 bit, and step size: $6 \mathrm{~nm}$. White arrows on gold nanoparticles correspond to individual ED patterns, Spacer $200 \mathrm{~nm}, 30 \mathrm{~nm} \mathrm{Si} \mathrm{N}_{4}$ thickness.

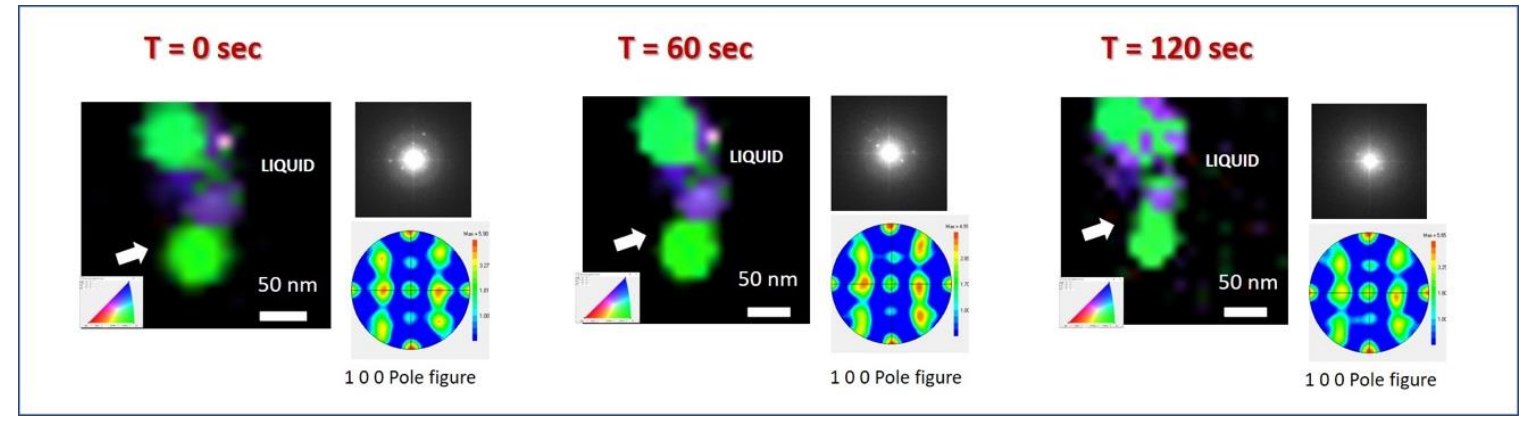

Figure 2. ASTAR orientation map of $220 \mathrm{~nm}$ x $220 \mathrm{~nm}$ (scan time $20 \mathrm{sec}$ ) by Medipix III detector, 20 fps, 12 bit, and step size: $11 \mathrm{~nm}$. White arrows on gold nanoparticles correspond to individual ED patterns, Spacer $200 \mathrm{~nm}, 30 \mathrm{~nm} \mathrm{Si}{ }_{3} \mathrm{~N}_{4}$ thickness.

\section{References}

[1] J. Portillo, E. F. Rauch, S. Nicolopoulos, M. Gemmi, and D. Bultreys. "Precession electron diffraction assisted orientation mapping in the transmission electron microscope", Materials Science Forum, 644, 1$7,2010$.

[2] T. Welling, S. Sadighikia, K. Watanabe, A. C. Carbonell, M. Bransden, D. Nagao, A. V. Blaaderen and M. A. Huis. "Observation of undamped 3D Brownian motion of nanoparticles using Liquid cell scanning transmission microscopy" Part. Part. Syst. Charact, 37, 2000003, 2020. 